CASE REPORT

\title{
CD20 positive T cell lymphoma: is it a real entity?
}

\author{
T Sun, A Akalin, M Rodacker, T Braun
}

J Clin Pathol 2004;57:442-444. doi: 10.1136/icp.2003.011734

CD20+ T cell lymphoma is a rare condition that includes both precursor and peripheral T cell types. However, these cases may be mistaken for T cell marker positive B cell lymphoma, thus creating diagnostic problems with subsequent clinical implications. This report describes a unique case of nodal CD20+ T cell lymphoma with simultaneous cutaneous and subcutaneous involvement. Both nodal and cutaneous lymphomas demonstrated identical monoclonal bands in the T cell receptor rearrangement assay. However, no CD20 expression was demonstrated in the skin lesion. The instability of the CD20 antigen in this tumour may imply that CD20 is not an integral part of the tumour immunophenotype and probably plays no important role in its clinical course. Techniques to avoid misdiagnosis of this lymphoma are discussed.

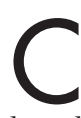
oexpression of CD20 and a T cell marker (such as CD43 or CD5) is commonly encountered in low grade B cell lymphomas and is useful in the distinction between lymphomas and reactive B cell proliferation. ${ }^{1}$ However, there are rare cases of $\mathrm{T}$ cell lymphomas that coexpress CD20 with $\mathrm{T}$ cell markers. ${ }^{1-9}$ Therefore, if only a small immunohistochemical panel-such as CD20, CD43, and CD5-is used, it cannot distinguish a CD5+/CD43+ B cell lymphoma from a CD20+ $\mathrm{T}$ cell lymphoma. ${ }^{2}$ The clinical implications of misdiagnosing a $\mathrm{T}$ cell lymphoma as a B cell lymphoma can be very serious. For this entity, the issues are how to avoid this misdiagnosis and the clinical relevance of CD20 expression in a $\mathrm{T}$ cell lymphoma. We report a case of CD20+ T cell lymphoma in a lymph node with the absence of CD20 expression in the cutaneous lymphomatous lesion.

"The clinical implications of misdiagnosing a T cell lymphoma as a B cell lymphoma can be very serious"

\section{CASE REPORT}

An 80 year old man presented with swelling, redness, and tenderness over the left breast and axilla for four weeks. Physical examination showed pronounced induration of the skin below the left breast and extending into the left axilla. Several lymph nodes measuring $5-6 \mathrm{~cm}$ in aggregate were noted in the left axilla. Computerised tomography scans demonstrated bilateral axillary lymphadenopathy and soft tissue inflammation in the skin and subcutaneous tissue inferior to the left axilla. Bulky inguinal adenopathy was present bilaterally. Axillary lymph node and skin biopsies were performed.

\section{Pathological findings}

The axillary lymph node showed complete effacement of the normal architecture by infiltration of large atypical pleomorphic lymphoid cells on a diffuse sclerotic background.
The tumour cells had finely stippled nuclear chromatin with occasional nucleoli and a moderate amount of amphophilic cytoplasm (fig 1). The mitotic rate was high. Some areas revealed inflammatory infiltration including mature lymphocytes, plasma cells, and eosinophils.

The skin biopsy showed atypical lymphoid cell infiltrates similar to those seen in the lymph node biopsy. The infiltration was concentrated mainly in the upper dermis, but lower dermis, epidermis, and subcutaneous adipose tissue were also mildly involved.

\section{Immunophenotyping}

Flow cytometric analysis of the lymph node revealed that the neoplastic cells were positive for all T cell markers (CD2, CD3, CD4, CD5, and CD8) except for CD7. For B cell markers, the cells were positive for CD20 and FMC-7, but negative for CD79a. Surface and cytoplasmic immunoglobulins were not expressed and terminal deoxynucleotidyl transferase (TdT) was negative.

The initial immunohistochemical stains of the lymph node showed positive reactions to CD3 (fig 2), CD45RO, and CD20 (fig 3). Repeat immunohistochemical staining at the Mayo Clinic revealed positive staining for CD3 (strong), CD45, and CD20 (weak). The tumour cells were also focally positive for CD30 but not for CD15. Further studies at the Armed Forces Institute of Pathology (AFIP) showed that the tumour cells were positive for CD3, CD4, CD5, CD8 (weak), and CD20, and partially positive for CD30. There was no staining for CD7, CD10, CD56, CD79a, ALK-1, PAX5, TIA-1, granzyme, or latent membrane protein 1 of the Epstein Barr virus.

Immunohistochemical staining of the skin biopsy showed that the tumour cells were positive for CD3 and CD45RO, but not for CD20.

\section{Molecular study}

Polymerase chain reaction was performed on the lymph node specimen at the AFIP and showed monoclonal bands in both the T cell receptor (TCR) $\gamma$ chain gene and the TCR $\beta$ chain gene rearrangement assays. The skin biopsy also revealed monoclonal TCR $\gamma$ chain gene rearrangement, and the biallelic monoclonal bands at 71 and $78 \mathrm{bp}$ were identical to those seen in the lymph node biopsy. Immunoglobulin heavy chain gene rearrangement was not demonstrated in the lymph node specimen.

\section{DISCUSSION}

The presence of TCR $\gamma$ and $\beta$ chain gene rearrangements and the positive reactions for CD3, CD4, CD5, CD8, and CD45RO indicate that this tumour is a T cell lymphoma. The positivity for CD20 in this tumour represents an aberrant immunophenotype, but it is not a B cell lymphoma, as shown by the negative immunoglobulin heavy chain gene rearrangement,

Abbreviations: AFIP, Armed Forces Institute of Pathology; TCR, T cell receptor; TdT, terminal deoxynucleotidyl transferase 


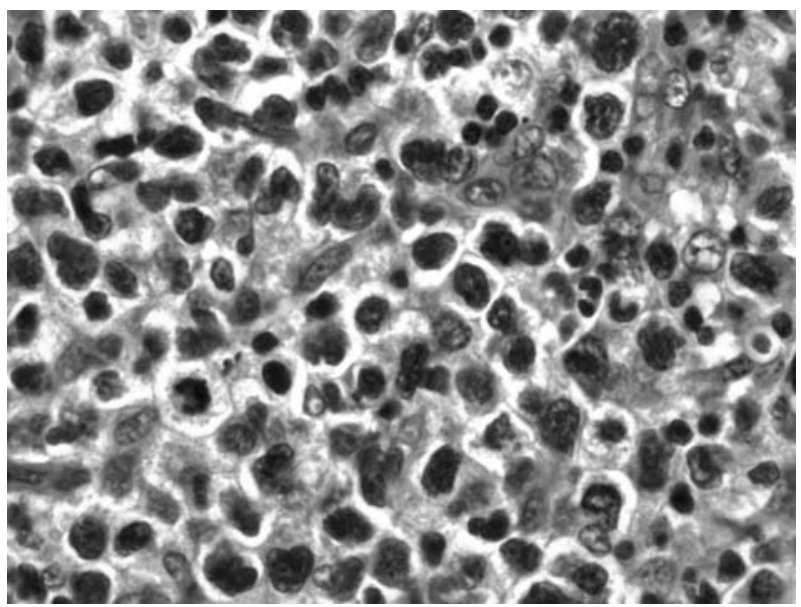

Figure 1 The lymph node biopsy shows large, pleomorphic lymphoma cells with irregular nuclear configuration and occasional nucleoli. A few mitotic figures are seen. Haematoxylin and eosin stain.

and the absence of other B cell markers, such as CD79a and PAX5. The negative reactions for TdT and CD10 exclude precursor $\mathrm{T}$ cell lymphoma. Although some tumour cells expressed CD30, the morphology of the tumour and the negative reactions for ALKl, TIA-1, and granzyme are not supportive of anaplastic large cell lymphoma. The absence of CD56, TIA-1, and granzyme reactivity excludes a natural killer/T cell lymphoma.

Expression of CD20 has been reported in both precursor and peripheral T cell lymphomas. ${ }^{1}$ Although it is not unusual to see lineage infidelity in precursor lymphoid neoplasms, ${ }^{45}$ the expression of CD20 or another B cell marker, CD79a, in peripheral $\mathrm{T}$ cell lymphomas has caused some confusion in the lineage identification of lymphomas. Blakolmer et al reported the expression of CD79a in four cases and CD20 in one case of T cell lymphoma. ${ }^{6}$ These authors concluded that the expression of CD79a and CD20 in T cell lymphomas is mutually exclusive. However, a case of peripheral $\mathrm{T}$ cell lymphoma with coexpression of both CD20 and CD79a has been reported recently. ${ }^{4}$

The major issue in these studies is the use of a small panel of monoclonal antibodies for immunophenotyping. Algino et al expressed their concern about using CD5 and CD20 alone to analyse lymphoproliferative disorders, which may result in

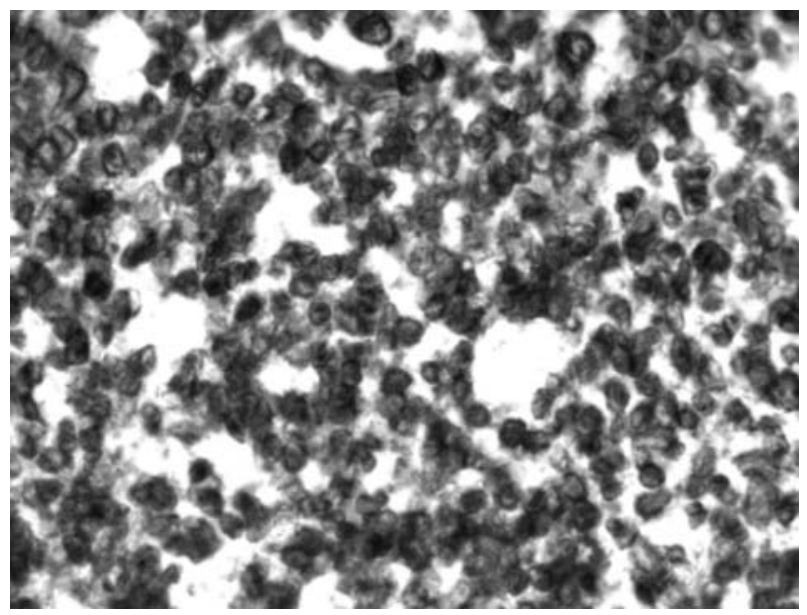

Figure 2 The tumour cells show extensive, strong CD3 staining. Immunoperoxidase stain.

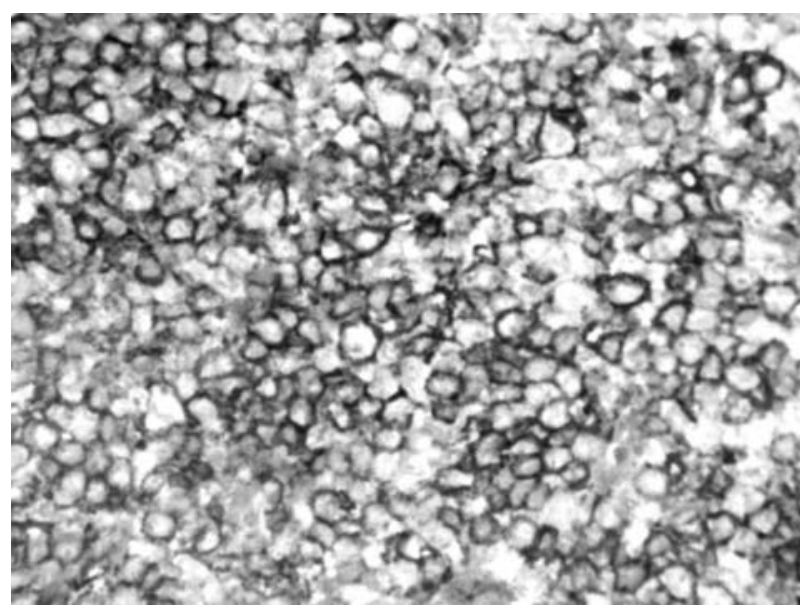

Figure 3 The tumour cells are also positive for CD20.

Immunoperoxidase stain.

confusion between CD5 expressing B cell lymphoma and CD20 expressing $\mathrm{T}$ cell lymphoma. ${ }^{2}$ Similarly, the use of a CD20-CD43 combination may lead to the misdiagnosis of a CD20+ T cell lymphoma as a B cell lymphoma. ${ }^{1}$

"The inclusion of CD19 in the flow cytometric panel may help to distinguish a B cell from a T cell lymphoma"

To avoid misdiagnosis, a large immunohistochemical panel was suggested by Blakolmer et al. ${ }^{6}$ However, we feel that flow cytometry is superior to immunohistochemistry in identifying $\mathrm{B}$ cell marker positive $\mathrm{T}$ cell lymphomas, because flow cytometry can demonstrate a clear cut dual staining of $\mathrm{B}$ cell and $\mathrm{T}$ cell markers. The intensity of CD20 fluorescent staining can also help to distinguish these two entities: CD20+ T cells stain strongly for CD5 and weakly for CD20, whereas CD5+ B cells show strong CD20 staining and weak CD5 staining. ${ }^{7}$ Furthermore, CD19 is frequently demonstrated in B cell lymphomas by flow cytometry, but is not available for immunohistochemistry. The inclusion of CD19 in the flow cytometric panel may help to distinguish a B cell from a T cell lymphoma.

\section{Take home messages}

- Rare cases of CD20+ T cell lymphoma can be mistaken for T cell marker positive B cell lymphoma, thus creating diagnostic problems, with subsequent clinical implications

- We describe a unique case of nodal CD20+ T cell lymphoma with simultaneous cutaneous and subcutaneous involvement where both nodal and cutaneous tumours had identical monoclonal bands in the T cell receptor rearrangement assay

- However, CD20 was not expressed in the skin lesion, so that this antigen may not be an integral part of the tumour immunophenotype and probably does not play an important role in its clinical course

- To avoid misdiagnosis, flow cytometry is preferable to immunohistochemistry and a large panel of antibodies should be used 
The absence of CD20 in the cutaneous lesion of our patient may imply that CD20 is not an integral part of this tumour. Similarly, Blakolmer et al reported that CD79a became negative in the second biopsies of two patients with CD79a+ T cell lymphoma. ${ }^{6}$ These authors suggested that the inconstant reactivity of CD79a might indicate crossreactivity with an unknown epitope(s). Because CD20 was positive on endothelial cells, macrophages, and epithelial cells in one study, the CD20 reaction might also be a non-specific binding of T cells. ${ }^{8}$ In spite of the presence of a small population of CD20+ T cells in healthy donors, ${ }^{3}$ the instability of CD20 in T cell tumours is not in favour of a malignant transformation of this population to lymphoma.

Takami et al suggested that CD20 might be an activation marker of $\mathrm{T}$ cells because the CD20+ $\mathrm{T}$ cells in their patient expressed several activation antigens, and monkey lymph node T cells weakly expressed CD20 after in vitro activation. ${ }^{5}$ Therefore, the expression of CD20 in T cell lymphoma could be the result of $\mathrm{T}$ cell activation. This hypothesis may explain why staining for CD20 is absent in the cutaneous lesion of our patient. Echeverri et al believed that the stability of an antigen is related to its biology and function. ${ }^{10}$ In this context, the B cell markers in a T cell lymphoma probably do not play an important role in the behaviour of the tumour.

Alternatively, the expression of CD20 in one tissue but not in another could represent clonal evolution, non-specific staining, or loss of CD20 peripherally. In any event, CD20+ T cell lymphoma may not be a real entity. However, a correct answer awaits further clinical studies.

\section{ACKNOWLEDGEMENTS}

This case was consulted with Drs C Barekman, J Taukenberger, and J Hallman, of the Armed Forces Institute of Pathology, and Dr ED Remstein, of the Mayo Clinic, USA.
Authors' affiliations

T Sun, T Braun, Veterans Affairs Medical Center and Department of Pathology, University of Colorado School of Medicine, Colorado, USA A Akalin, Department of Medicine, University of Colorado School of Medicine

M Rodacker, Anapath Diagnostics Inc, Cheyenne, Wyoming 82001, USA

Correspondence to: $\operatorname{Dr} T$ Sun, Pathology and Laboratory Medicine Service, VA Medical Center, 1055 Clermont St, Denver, CO 80220, USA; Tsieh.Jack.Sun@med.va.gov

Accepted for publication 16 September 2003

\section{REFERENCES}

1 Mohrmann RL, Arber DA. CD20-positive peripheral T-cell lymphoma: report of a case after nodular sclerosis Hodgkin's disease and review of the literature. Mod Pathol 2000;13:1244-52.

2 Algino KM, Thomason RW, King DE, et al. CD2O (pan-B cell antigen) expression on bone marrow-derived T cells. Am J Clin Pathol 1996;106:78-81.

3 Quintanilla-Martinez L, Preffer F, Rubin D, et al. CD20+ T-cell lymphoma: neoplastic transformation of a normal T-cell subset. Am J Clin Pathol 1994; 102:483-9.

4 Yao X, Teruya-Feldstein J, Raffeld M, et al. Peripheral T-cell lymphoma with aberrant expression of CD79a and CD20: a diagnostic piffall. Mod Pathol 2001;14:105-10

5 Takami A, Saito M, Nakao S, et al. CD20-positive T-cell chronic lymphocytic leukaemia. Br J Haematol 1998;102:1327-9.

6 Blakolmer K, Vesely M, Kummer JA, et al. Immunoreactivity of B-cell markers (CD79a, L26) in rare cases of extranodal cytotoxic peripheral T- (NK/T-) cell lymphomas. Mod Pathol 2000;13:766-72.

7 Hultin LE, Hausner MA, Hutlin PM, et al. CD20 (pan-B cell) antigen is expressed at a low level on a subpopulation of human T-lymphocytes. Cytometry 1993;14:196-204.

8 Hamilton-Dutoit SJ, Pallesen G. B-cell associated monoclonal antibody L26 may occasionally label T cell lymphoma. APMIS 1989:97:1033-6.

9 Warzynski MJ, Graham DM, Axtell RA, et al. Low level CD20 expression on T cell malignancies. Cytometry 1994; 18:88-92.

10 Echeverri C, Fisher S, King D, et al. Immunophenotypic variability of B-cell non-Hodgkin lymphoma. Am J Clin Pathol 2002;117:615-20. 